\title{
A proposed counselling attribute framework against game overuse
}

\begin{abstract}
In the past decade, online games problematic use such as excessive play and addiction have received tremendous attention not only from the media, but also from psychiatrists, psychologists, mental health associations and gamers alike. To address this issue, game developers have proposed to add warning messages about risk of overuse on the loading screens of certain online games. However, there is yet no evidence that such warning messages have made any serious impact on gamers. The current game design issues, proposed control measures against game overuse and game design frameworks were carefully examined. The objective of this paper is to propose a Counselling Attributes (CA) framework that will address the problematic and addictiveness of online games by adopting streaming counselling/warning messages at every 2 minutes in game design. This approach will increase the awareness of the problems online games possess more closely to gamers. As a result, excessive play, addiction and number of hours spent playing online games will drastically reduce.
\end{abstract}

Keyword: Counseling attribute framework; Game design; Game control; MDA; The 6-11 